\title{
The Relationship between Morphological and Functional Features of Preterm Placentas and the Results of Bacteriological Examination of the Discharge from the Cervical Canal of Women with Preterm Birth
}

\author{
Agamurad A. Orazmuradov, $\mathrm{PhD}, \mathrm{ScD}^{1}$; Igor N. Kostin, $\mathrm{PhD}, \mathrm{ScD}^{1}$; Marina B. Khamoshina, \\ $\mathrm{PhD}, \mathrm{ScD}^{1}$; Sergey V. Apresyan, $\mathrm{PhD}, \mathrm{ScD}^{1}$; Setonde Romeo D. Konnon, $\mathrm{PhD}^{1}$; Alexandra A. \\ Gavrilova, PGS ${ }^{1}$; Anna N. Parygina, $\mathrm{PGS}^{1}$; Gayane A. Arakelyan, $\mathrm{PGS}^{1}$; \\ Irina V. Savenkova, $\mathrm{PGS}^{1}$; Aleksey A. Lukaev, $\mathrm{PhD}^{2 *}$ \\ ${ }^{1}$ RUDN University, Moscow, Russia \\ ${ }^{2}$ Mytishchi City Clinical Hospital Mytishchi, Moscow Region, Russia
}

\begin{abstract}
The aim of this study was to determine the relationship between the morpho-functional features of placentas from preterm births (PBs) with the results of bacteriological examination of the discharge from the cervical canal in women with spontaneous preterm birth (SPB). The study included 227 pregnant women at gestational age between 28 and 36 weeks and 6 days, who underwent examination in the period from 2017 to 2018. Depending on the gestational age, they were divided into 3 groups. In general, a strong relationship was found between the presence of pathological changes in placentas and the presence of opportunistic flora in women ( $\mathrm{R}=0.722, P<0.001)$. The constructed mathematical models make it possible to determine, with a high degree of certainty, the main bacteria for all the studied groups of pregnant women, thereby identifying the risk group of women at the stage of pregnancy planning, predicting complications and increasing the possibility of deliver a full term baby. (International Journal of Biomedicine. 2019;9(4):313-319.)
\end{abstract}

Key Words: preterm birth $\bullet$ placenta $\bullet$ opportunistic flora $\bullet$ placental insufficiency

\section{Introduction}

Preterm birth $(\mathrm{PB})$ is a multifactorial syndrome with a variety of risk factors and long-term health consequences for the child. ${ }^{(1-3)}$ Every year, an estimated 15 million babies are born preterm (before 37 completed weeks of gestation), and this number is rising. ${ }^{(3)}$ Approximately 1 million children die each year due to complications of $\mathrm{PB} \cdot{ }^{(4)}$ Common causes of PB include multiple pregnancies, infections and chronic conditions such as diabetes and high blood pressure. ${ }^{(3)}$

There is a large body of evidence that a cascade of activations of cellular components and mediators of inflammatory pathways results in onset of labor and membrane rupture. ${ }^{(5,6)}$ Supporting the fetus through the preceding gestation, the placenta is a very critical

"Corresponding author: Aleksey A. Lukaev, PhD. Mytishchi municipal clinical hospital, Mytishchi, Moscow Region, Russia E-mail: alekseilukaev@mail.ru organ in explaining the pathogenesis of spontaneous preterm birth (SPB). Placental pathology provides important diagnostic information to ascertain the cause of PB. Intra-amniotic infection, as is well known, is one risk factor of SPB. ${ }^{(6-8)}$ Bacterial infection and the subsequent inflammatory response are recognized as an important cause of PB. It is hypothesized that these organisms ascend the cervical canal, colonize placental tissues, cause chorioamnionitis and in severe cases infect amniotic fluid and the fetus. ${ }^{(9)}$ However, after 32 weeks' gestation, infection may be a less common cause of PB; instead, many cases of spontaneous preterm labor leading to $\mathrm{PB}$ appear to be caused by placental insufficiency (PI). ${ }^{(1)}$ The pathoanatomical investigation of placentas from PBs is useful for assessing the etiology of SPB and the prognosis for the child. ${ }^{(10)}$

The aim of this study was to determine the relationship between the morpho-functional features of placentas from PBs with the results of bacteriological examination of the discharge from the cervical canal in women with SPB. 


\section{Materials and Methods}

Our study included 227 pregnant women at gestational age between 28 and 36 weeks and 6 days, who underwent examination in the period from 2017 to 2018. Depending on the gestational age, they were divided into 3 groups. Group 1 included 73 women at gestational age between 28 and 30 weeks and 6 days; Group 2 included 81 women at gestational age between 31 and 33 weeks and 6 days, Group 3 included 73 women at gestational age between 34 and 36 weeks and 6 days.

All women underwent an assessment of vaginal microcenosis and the quantitative and qualitative composition of the biotope of the cervical discharge, as we described earlier. ${ }^{(8)}$

The examination of placentas was carried out according to a standardized scheme, including macroscopic analysis, material sampling and histological analysis in 3 stages. At Stage 1 of the histological examination, we performed an assessment of placental maturity; ${ }^{(11)}$ at Stage 2 - a semiquantitative assessment of the severity of certain structural indicators of the placenta (from 1 to 3 points); and at Stage 3 - the determination of the degree of PI by the totality of the mass of the fetus and placenta, the degree of maturity or immaturity of the villi, the severity of compensatory reactions, and involutive changes.

The study was conducted in accordance with ethical principles of the WMA Declaration of Helsinki (1964, ed. 2013) and approved by the RUDN University Ethics Committee. Written informed consent was obtained from all participants.

Statistical analysis was performed using the Statistica 8.0 software package (StatSoft Inc, USA). Baseline characteristics were summarized as frequencies and percentages for categorical variables and as mean \pm SEM for continuous variables. MannWhitney U test and Kruskal-Wallis test were used, respectively, to compare means of 2 and 3 or more groups of variables not normally distributed. The frequencies of categorical variables were compared using Pearson $\chi^{2}$ or Fisher's exact test, when appropriate. The Pearson's correlation coefficient and Spearman's rank correlation coefficient were was used to determine the strength and direction of the relationship between two variables. The odds ratio (OR), its standard error and $95 \%$ confidence interval (CI) were calculated. Logistic regression was used to model dichotomous outcome variables. We calculated the sensitivity, specificity, positive predictive values, negative predictive values, and accuracy of the test results. A value of $P<0.05$ was considered statistically significant.

\section{Results}

The average weight of placentas in Groups 1, 2 and 3 was $237.3 \pm 49.1 \mathrm{~g}, 228.3 \pm 94.1 \mathrm{~g}$, and $318.1 \pm 90.5 \mathrm{~g}$, respectively (Kruskal Wallis test: $\mathrm{H}=18.30641, P=0.0001$ ). Macroscopically, placentas had an ovoid or irregular shape, small size, with additional bulges, more thickened in the center, with thinning at the edges. The umbilical cord was attached at the edges (paraplacental), in single observations - in the central zone. The length of the umbilical cord was in the range from $40 \mathrm{~cm}$ to $68 \mathrm{~cm}$ (an average of $46 \pm 0.6 \mathrm{~cm}$ ). It was noted that the vessels passing through the umbilical cord had a less than normal

winding and spiral course. The fetal surface of placentas was smooth, individual placentas had whitish areas. The maternal surface had smoothed or flattened cotyledons. In all studies, fine-grained calcifications were noted. Morphological changes in placentas are presented in Figures 1-8.

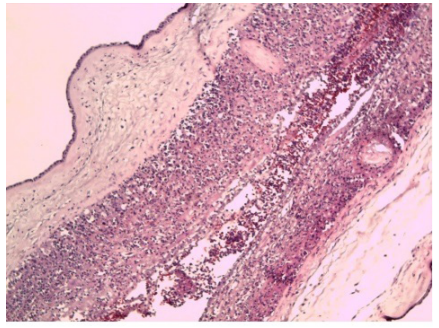

Fig.1. Leukocyte infiltration in placentas (H\&E staining, $x 400)$.

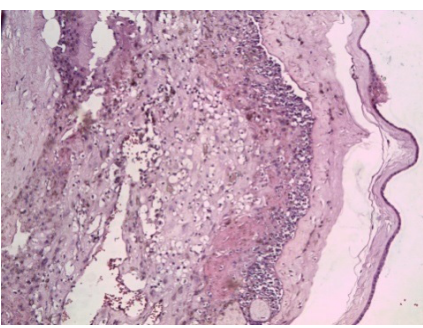

Fig.3. Signs of inflammation in the amnion (H\&E staining, $x 400)$.

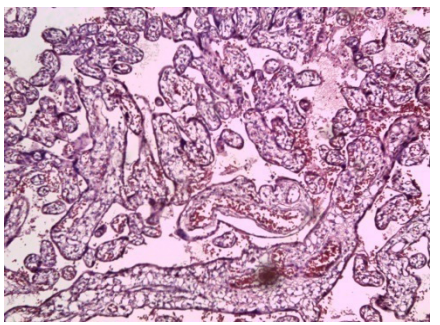

(A)

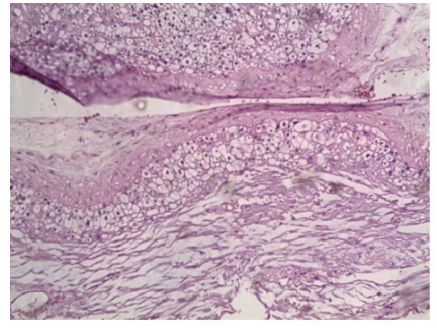

Fig.2. Signs of productive inflammation (H\&E staining, x400)

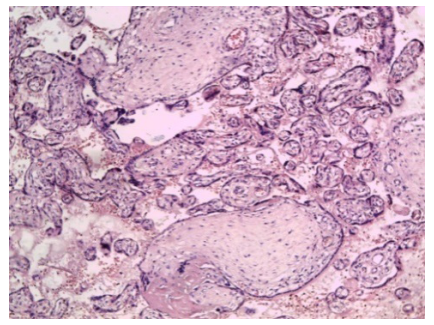

Fig.4. Intermediate chorionic villi with a small number of capil-laries, sometimes surrounded by fibrinoid masses (H\&E staining, x400).

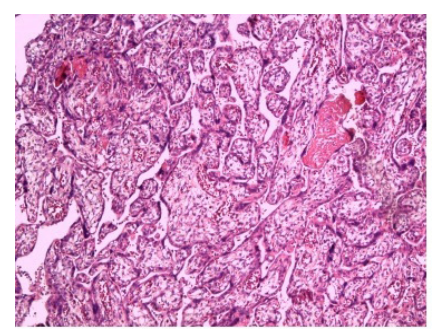

(B)
Fig.5 (A,B). Intermediate chorionic villi (the 1st and 2nd ramifications). In many fields of view, the capillaries of the supporting and intermediate villi are "empty" (H\&E staining; x400)

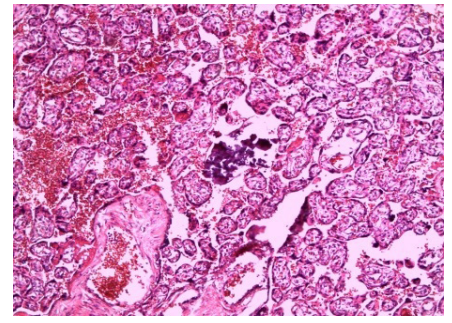

(A)

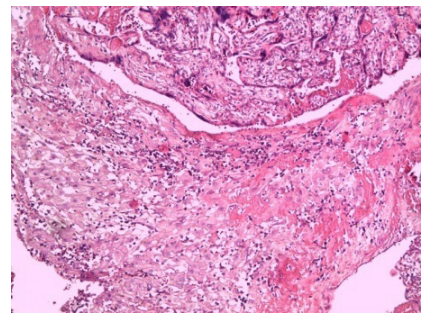

(B)
Fig.6 $(\boldsymbol{A}, \boldsymbol{B})$. Diffuse inflammation with foci of calcareous deposits in the form of calcifications in decidual tissue.

In some places, the immature terminal chorionic villi are closely adjacent to each other, therefore, the intervillous space is weakly expressed. The chorionic villi are partially surrounded by a fibrinoid roller. There is plethora of immature villi and their "desolation" in some places. In the intervillosis space, erythrocyte "fields" are noted, as well as foci of calcareous deposits in the form of calcifications (H\&E staining, $x$ 400). 


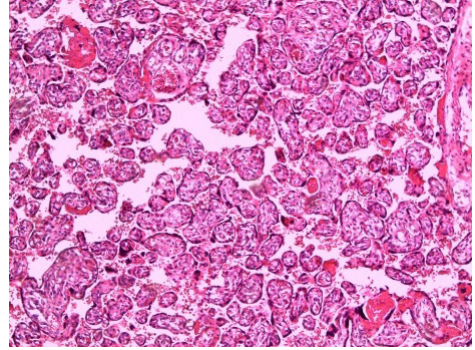

Fig.7. Pathological immaturity of terminal chorionic villi (H\&E staining, $x$ 400).

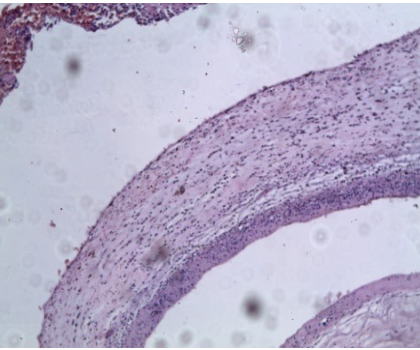

(A)

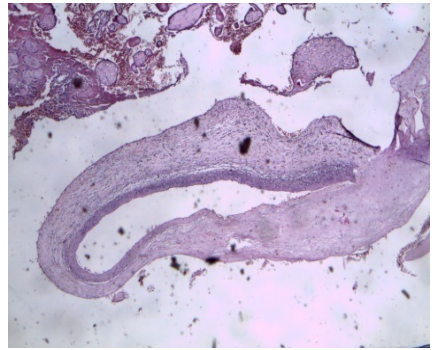

(B)
Fig.8. Choriodeciduite. Severe leukocyte infiltration within the dicidal and trophoblastic layers. Moderate edema of spongy space. (H\&E staining; $A-x 400, B-x 200)$.

The data from the correlation analysis of the relationship between the state of the placenta and the data from bacteriological examination of the cervical discharge are presented in Tables 1-9.

\section{Table 1.}

Placentas with histological signs of inflammation and necrotic changes in Group 1

\begin{tabular}{|l|c|c|c|c|c|}
\hline \multicolumn{1}{|c|}{ Group 1 } & $\begin{array}{c}\text { Signs }+ \\
\mathrm{n}=36\end{array}$ & $\begin{array}{c}\text { Signs - } \\
\mathrm{n}=37\end{array}$ & $P$ & $\begin{array}{c}\text { OR } \\
(95 \% \mathrm{CI})\end{array}$ & $\mathrm{R}$ \\
\hline E.coli & $6(16.7 \%)$ & $12(32.4 \%)$ & 0.1153 & $0.51(0.22-1.22)$ & \\
\hline Candida spp & $4(11.1 \%)$ & $6(16.2 \%)$ & 0.5259 & $0.69(0.21-2.23)$ & \\
\hline $\begin{array}{l}\text { Enterococcus } \\
\text { faecalis }\end{array}$ & $15(41.7 \%)$ & $12(32.4 \%)$ & 0.4139 & $1.28(0.7-2.35)$ & \\
\hline Str. agalactiae & $10(27.8 \%)$ & $3(8.1 \%)$ & 0.0281 & $3.43(1.03-11.44)$ & $\begin{array}{c}0.472 \\
P=0.003\end{array}$ \\
\hline $\begin{array}{l}\text { Staph. } \\
\text { epidermidis }\end{array}$ & $10(27.8 \%)$ & $4(10.8 \%)$ & 0.0656 & $2.57(0.89-7.45)$ & $\begin{array}{c}0.307 \\
P=0.042\end{array}$ \\
\hline Staph. aureus & $3(8.3 \%)$ & $4(10.8 \%)$ & 0.7193 & $0.77(0.19-3.2)$ & \\
\hline $\begin{array}{l}\text { Staph. } \\
\text { haemolyticus }\end{array}$ & $8(22.2 \%)$ & $2(5.4 \%)$ & 0.0367 & $4.11(0.94-18.06)$ & $\begin{array}{c}0.328 \\
P=0.029\end{array}$ \\
\hline $\begin{array}{l}\text { Enterobacter } \\
\text { aerogenes }\end{array}$ & $9(25 \%)$ & $2(5.4 \%)$ & 0.0193 & $4.63(1.07-19.95)$ & $\begin{array}{c}0.472 \\
P=0.003\end{array}$ \\
\hline Acinebacter & $10(27.8 \%)$ & $3(8.1 \%)$ & 0.0281 & $3.43(1.03-11.44)$ & $\begin{array}{c}0.603 \\
P<0.001\end{array}$ \\
\hline Str. viridans & $3(8.3 \%)$ & $6(16.2 \%)$ & 0.3057 & $0.51(0.14-1.9)$ & \\
\hline Staph. warneri & $5(13.9 \%)$ & $7(18.9 \%)$ & 0.5621 & $0.73(0.26-2.1)$ & \\
\hline $\begin{array}{l}\text { Serratia } \\
\text { marcescens }\end{array}$ & $3(8.3 \%)$ & $5(13.5 \%)$ & 0.4787 & $0.62(0.16-2.39)$ & \\
\hline
\end{tabular}

Table 2.

Placentas with histological signs of pathological immaturity in Group 1

\begin{tabular}{|l|c|c|c|c|c|}
\hline \multicolumn{1}{|c|}{ Group 1 } & $\begin{array}{c}\text { Signs }+ \\
\mathrm{n}=38\end{array}$ & $\begin{array}{c}\text { Signs- } \\
\mathrm{n}=35\end{array}$ & $P$ & $\begin{array}{c}\text { OR } \\
(95 \% \mathrm{CI})\end{array}$ & $\mathrm{R}$ \\
\hline E.coli & $6(15.8 \%)$ & $12(34.3 \%)$ & 0.0670 & $0.46(0.19-1.09)$ & \\
\hline Candida spp. & $4(10.5 \%)$ & $6(17.1 \%)$ & 0.4114 & $0.61(0.19-2)$ & \\
\hline $\begin{array}{l}\text { Enterococcus } \\
\text { faecalis }\end{array}$ & $20(52.6 \%)$ & $7(20 \%)$ & 0.0039 & $2.63(1.27-5.45)$ & $\begin{array}{c}0.472 \\
P=0.003\end{array}$ \\
\hline Str. agalactiae & $10(23.7 \%)$ & $3(8.6 \%)$ & 0.0818 & $2.76(0.81-9.39)$ & $\begin{array}{c}0.307 \\
P=0.042\end{array}$ \\
\hline $\begin{array}{l}\text { Staph. } \\
\text { epidermidis }\end{array}$ & $11(28.9 \%)$ & $3(8.6 \%)$ & 0.0272 & $3.38(1.03-11.11)$ & $\begin{array}{c}0.328 \\
P=0.029\end{array}$ \\
\hline Staph. aureus & $3(7.9 \%)$ & $4(11.4 \%)$ & 0.6085 & $0.69(0.17-2.87)$ & \\
\hline $\begin{array}{l}\text { Staph. } \\
\text { haemolyticus }\end{array}$ & $3(7.9 \%)$ & $7(20 \%)$ & 0.1329 & $0.39(0.11-1.41)$ & \\
\hline $\begin{array}{l}\text { Enterobacter } \\
\text { aerogenes }\end{array}$ & $5(13.2 \%)$ & $6(17.1 \%)$ & 0.6345 & $0.77(0.26-2.29)$ & \\
\hline Acinebacter & $10(26.3 \%)$ & $3(8.6 \%)$ & 0.0477 & $3.07(0.92-10.25)$ & $\begin{array}{c}0.405 \\
P=0.008\end{array}$ \\
\hline Str. viridans & $8(21.1 \%)$ & $1(2.9 \%)$ & 0.0182 & $7.37(0.97-55.97)$ & $\begin{array}{c}0.419 \\
P=0.005\end{array}$ \\
\hline Staph. warneri & $4(10.5 \%)$ & $8(22.9 \%)$ & 0.1556 & $0.46(0.15-1.4)$ & \\
\hline $\begin{array}{l}\text { Serratia } \\
\text { marcescens }\end{array}$ & $3(7.9 \%)$ & $5(14.3 \%)$ & 0.3825 & $0.55(0.14-.14)$ & \\
\hline
\end{tabular}

Table 3.

Placentas with histological signs of placental insufficiency and hypertension in Group 1

\begin{tabular}{|l|c|c|c|c|c|}
\hline \multicolumn{1}{|c|}{ Group 1 } & $\begin{array}{c}\text { Signs } \\
\mathrm{n}=28\end{array}$ & $\begin{array}{c}\text { Signs - } \\
\mathrm{n}=45\end{array}$ & $P$ & $\begin{array}{c}\text { OR } \\
(95 \% \mathrm{CI})\end{array}$ & $\mathrm{R}$ \\
\hline E.coli & $12(42.9 \%)$ & $6(13.3 \%)$ & 0.0070 & $3.21(1.36-7.59)$ & $\begin{array}{c}0.378 \\
P=0.008\end{array}$ \\
\hline Candida spp. & $9(32.1 \%)$ & $1(2.2 \%)$ & 0.0003 & $14.46(1.94-108.11)$ & $\begin{array}{c}0.405 \\
P=0.004\end{array}$ \\
\hline $\begin{array}{l}\text { Enterococcus } \\
\text { faecalis }\end{array}$ & $15(53.6 \%)$ & $12(26.7 \%)$ & 0.0206 & $2.01(1.11-3.64)$ & $\begin{array}{c}0.326 \\
P=0.031\end{array}$ \\
\hline $\begin{array}{l}\text { Staph. } \\
\text { haemolyticus }\end{array}$ & $7(25 \%)$ & $6(13.3 \%)$ & 0.2295 & $1.88(0.70-5.01)$ & \\
\hline $\begin{array}{l}\text { Staph. } \\
\text { epidermidis }\end{array}$ & $9(32.1 \%)$ & $5(11.1 \%)$ & 0.0265 & $2.89(1.08-7.76)$ & $\begin{array}{c}0.339 \\
P=0.027\end{array}$ \\
\hline $\begin{array}{l}\text { Staph. } \\
\text { aureus }\end{array}$ & $5(17.9 \%)$ & $2(4.4 \%)$ & 0.0584 & $4.02(0.84-19.32)$ & $\begin{array}{c}0.386 \\
P=0.008\end{array}$ \\
\hline $\begin{array}{l}\text { Enterobacter } \\
\text { aerogenes }\end{array}$ & $9(32.1 \%)$ & $2(4.4 \%)$ & 0.0013 & $7.23(1.68-31.07)$ & $\begin{array}{c}0.563 \\
P<0.001\end{array}$ \\
\hline Acinebacter & $5(17.9 \%)$ & $8(17.8 \%)$ & 0.9931 & $1(0.36-2.77)$ & \\
\hline Str. viridans & $8(28.6 \%)$ & $1(2.2 \%)$ & 0.0009 & $12.86(1.7-97.37)$ & $\begin{array}{c}0.559 \\
P<0.001\end{array}$ \\
\hline $\begin{array}{l}\text { Staph. } \\
\text { warneri }\end{array}$ & $3(10.7 \%)$ & $9(20 \%)$ & 0.2979 & $0.54(0.16-1.81)$ & \\
\hline $\begin{array}{l}\text { Serratia } \\
\text { marcescens }\end{array}$ & $6(21.4 \%)$ & $2(4.4 \%)$ & 0.0239 & $4.82(1.04-22.25)$ & $\begin{array}{c}0.350 \\
P=0.014\end{array}$ \\
\hline
\end{tabular}


In Group 1, the number of placentas with signs of inflammation and necrotic changes was significantly higher in women with isolated Acinetobacter, Strep. agalactiae, Staph. haemolyticus, and Enterobacter aerogenes. The number of placentas with signs of pathological immaturity was higher in women with isolated Acinetobacter, Enterococcus faecalis, Staph. Epidermidis, and Str. Vridans. The number of placentas with signs of PI and hypertension was higher in women with isolated E. coli, Candida spp., Enterococcus faecalis, Staph. Epidermidis, Enterobacter aerogenes, Str. Viridans, and Serratia marcescens.

Table 4.

Placentas with histological signs of inflammation and necrotic changes in Group 2

\begin{tabular}{|l|c|c|c|c|c|}
\hline \multicolumn{1}{|c|}{ Group 2 } & $\begin{array}{c}\text { Signs }+ \\
\mathrm{n}=47\end{array}$ & $\begin{array}{c}\text { Signs }- \\
\mathrm{n}=34\end{array}$ & $P$ & $\begin{array}{c}\mathrm{OR} \\
(95 \% \mathrm{CI})\end{array}$ & $\mathrm{R}$ \\
\hline E.coli & $6(12.8 \%)$ & $9(26.5 \%)$ & 0.1171 & $0.48(0.19-1.23)$ & \\
\hline Candida spp. & $4(8.5 \%)$ & $8(23.5 \%)$ & 0.0604 & $0.36(0.12-1.1)$ & \\
\hline $\begin{array}{l}\text { Enterococcus } \\
\text { faecalis }\end{array}$ & $14(29.8 \%)$ & $4(11.8 \%)$ & 0.0542 & $2.53(0.91-7.02)$ & $\begin{array}{c}0.260 \\
P=0.024\end{array}$ \\
\hline $\begin{array}{l}\text { Str. } \\
\text { agalactiae }\end{array}$ & $6(12.8 \%)$ & $4(11.8 \%)$ & 0.8925 & $1.09(0.33-3.55)$ & \\
\hline $\begin{array}{l}\text { Staph. } \\
\text { epidermidis }\end{array}$ & $11(23.4 \%)$ & $4(11.8 \%)$ & 0.1832 & $1.99(0.69-5.72)$ & $\begin{array}{c}0.267 \\
P=0.033\end{array}$ \\
\hline Staph. aureus & $3(6.4 \%)$ & $6(17.6 \%)$ & 0.1114 & $0.36(0.1-1.35)$ & \\
\hline $\begin{array}{l}\text { Staph. } \\
\text { haemolyticus }\end{array}$ & $5(10.6 \%)$ & $7(20.6 \%)$ & 0.2135 & $0.52(0.18-1.49)$ & \\
\hline $\begin{array}{l}\text { Enterobacter } \\
\text { aerogenes }\end{array}$ & $8(17 \%)$ & $2(5.9 \%)$ & 0.1326 & $2.89(0.66-12.78)$ & $\begin{array}{c}0.257 \\
P=0.036\end{array}$ \\
\hline Acinebacter & $12(25.5 \%)$ & $2(5.9 \%)$ & 0.0210 & $4.34(1.04-18.15)$ & $\begin{array}{c}0.817 \\
P<0.001\end{array}$ \\
\hline Str. viridans & $8(17 \%)$ & $1(2.9 \%)$ & 0.0466 & $5.79(0.76-44.13)$ & $\begin{array}{c}0.484 \\
P<0.00\end{array}$ \\
\hline $\begin{array}{l}\text { Staph. } \\
\text { warneri }\end{array}$ & $10(21.3 \%)$ & $1(2.9 \%)$ & 0.0174 & $7.23(0.97-53.87)$ & $\begin{array}{c}0.491 \\
P<0.001\end{array}$ \\
\hline $\begin{array}{l}\text { Serratia } \\
\text { marcescens }\end{array}$ & $3(6.4 \%)$ & $3(8.8 \%)$ & 0.6789 & $0.72(0.16-3.37)$ & \\
\hline
\end{tabular}

In Group 2, the number of placentas with signs of inflammation and necrotic changes was significantly higher in women with isolated Str. Vridans, Acinetobacter, and Staph. Warneri. The number of placentas with signs of pathological immaturity was higher in women with isolated E. coli, Candida spp., Enterococcus faecalis, Staph. Epidermidis, and Acinetobacter. The number of placentas with signs of PI and hypertension was higher in women with isolated E. coli, Staph. aureus, Staph. haemolyticus, Acinetobacter, and Str. Viridans.

In Group 3, the number of placentas with signs of inflammation and necrotic changes was significantly higher in women with isolated Enterococcus faecalis and Staph. Warneri. The number of placentas with signs of pathological immaturity was higher in women with isolated Enterococcus faecalis and Staph. aureus. The number of placentas with signs of PI and hypertension was higher in women with isolated E. coli, Staph. aureus, and Acinetobacter.
Table 5.

Placentas with histological signs of pathological immaturity in Group 2

\begin{tabular}{|l|c|c|c|c|c|}
\hline \multicolumn{1}{|c|}{ Group 2 } & $\begin{array}{c}\text { Signs }+ \\
\mathrm{n}=48\end{array}$ & $\begin{array}{c}\text { Signs - } \\
\mathrm{n}=33\end{array}$ & $P$ & $\begin{array}{c}\mathrm{OP} \\
(95 \% \mathrm{CI})\end{array}$ & $\mathrm{R}$ \\
\hline E.coli & $13(27.1 \%)$ & $2(6.1 \%)$ & 0.0167 & $4.47(1.08-18.51)$ & $\begin{array}{c}0.319 \\
P=0.041\end{array}$ \\
\hline Candida spp. & $1(2.1 \%)$ & $11(33.3 \%)$ & 0.0001 & $0.06(0.01-0.46)$ & \\
\hline $\begin{array}{l}\text { Enterococcus } \\
\text { faecalis }\end{array}$ & $15(31.3 \%)$ & $3(9.1 \%)$ & 0.0184 & $3.44(1.08-10.94)$ & $\begin{array}{c}0.335 \\
P=0.001\end{array}$ \\
\hline $\begin{array}{l}\text { Strep. } \\
\text { agalactiae }\end{array}$ & $4(8.3 \%)$ & $6(18.2 \%)$ & 0.1855 & $0.46(0.14-1.5)$ & \\
\hline $\begin{array}{l}\text { Staph. } \\
\text { epidermidis }\end{array}$ & $13(27.1 \%)$ & $2(6.1 \%)$ & 0.0167 & $4.47(1.08-18.51)$ & $\begin{array}{c}0.527 \\
P<0.001\end{array}$ \\
\hline $\begin{array}{l}\text { Staph. } \\
\text { aureus }\end{array}$ & $8(16.7 \%)$ & $1(3 \%)$ & 0.0550 & $5.5(0.72-41.92)$ & $\begin{array}{c}0.493 \\
P<0.001\end{array}$ \\
\hline $\begin{array}{l}\text { Staph. } \\
\text { haemolyticus }\end{array}$ & $6(12.5 \%)$ & $6(18.2 \%)$ & 0.4794 & $0.69(0.24-1.95)$ & \\
\hline $\begin{array}{l}\text { Enterobacter } \\
\text { aerogenes }\end{array}$ & $7(14.6 \%)$ & $3(9.1 \%)$ & 0.4603 & $1.6(0.45-5.76)$ & \\
\hline Acinebacter & $12(25 \%)$ & $2(6.1 \%)$ & 0.0268 & $4.13(0.99-17.23)$ & $\begin{array}{c}0.607 \\
P<0.001\end{array}$ \\
\hline $\begin{array}{l}\text { Str. viridans } \\
\text { Staph. } \\
\text { warneri }\end{array}$ & $8(16.7 \%)$ & $1(3 \%)$ & 0.0550 & $5.5(0.72-41.92)$ & $\begin{array}{c}0.476 \\
P<0.001\end{array}$ \\
\hline $\begin{array}{l}\text { Serratia } \\
\text { marcescens }\end{array}$ & $2(4.2 \%)$ & $4(12.1 \%)$ & 0.1792 & $0.34(0.07-1.77)$ & \\
\hline
\end{tabular}

Table 6.

Placentas with histological signs of PI and hypertension in Group 2

\begin{tabular}{|c|c|c|c|c|c|}
\hline Group 2 & $\begin{array}{c}\text { Signs }+ \\
\mathrm{n}=44\end{array}$ & $\begin{array}{l}\text { Signs - } \\
\mathrm{n}=37\end{array}$ & $P$ & $\begin{array}{c}\mathrm{OP} \\
(95 \% \mathrm{CI})\end{array}$ & $\mathrm{R}$ \\
\hline E.coli & $14(31.8 \%)$ & $1(2.7 \%)$ & 0.0008 & $11.77(1.62-85.36)$ & $\begin{array}{c}0.793 \\
P<0.001\end{array}$ \\
\hline Candida spp. & $9(20.5 \%)$ & $3(8.1 \%)$ & 0.1192 & $2.52(0.74-8.64)$ & $\begin{array}{c}0.283 \\
P=0.051\end{array}$ \\
\hline $\begin{array}{l}\text { Enterococcus } \\
\text { faecalis }\end{array}$ & $1(2.3 \%)$ & $17(45.9 \%)$ & 0.0000 & $0.05(0.01-0.35)$ & \\
\hline Str. agalactiae & $4(9.1 \%)$ & $6(16.2 \%)$ & 0.3315 & $0.56(0.17-1.84)$ & \\
\hline $\begin{array}{l}\text { Staph. } \\
\text { epidermidis }\end{array}$ & $6(13.6 \%)$ & $9(24.3 \%)$ & 0.2174 & $0.56(0.22-1.43)$ & \\
\hline Staph. aureus & $8(18.2 \%)$ & $1(2.7 \%)$ & 0.0272 & $6.73(0.88-51.35)$ & $\begin{array}{c}0.317 \\
P=0.009\end{array}$ \\
\hline $\begin{array}{l}\text { Staph. } \\
\text { haemolyticus }\end{array}$ & $3(6.8 \%)$ & $9(24.3 \%)$ & 0.0272 & $0.28(0.08-0.96)$ & \\
\hline $\begin{array}{l}\text { Enterobacter } \\
\text { aerogenes }\end{array}$ & $8(18.2 \%)$ & $2(5.4 \%)$ & 0.0816 & $3.36(0.76-14.87)$ & $\begin{array}{c}0.276 \\
P=0.032\end{array}$ \\
\hline Acinebacter & $12(27.3 \%)$ & $2(5.4 \%)$ & 0.0095 & $5.05(1.21-21.12)$ & $\begin{array}{c}0.727 \\
P<0.001\end{array}$ \\
\hline Str. viridans & $8(18.2 \%)$ & $1(2.7 \%)$ & 0.0272 & $6.73(0.88-51.35)$ & \\
\hline $\begin{array}{l}\text { Staph. } \\
\text { warneri }\end{array}$ & $3(6.8 \%)$ & $8(21.6 \%)$ & 0.0527 & $0.32(0.09-1.1)$ & \\
\hline $\begin{array}{l}\text { Serratia } \\
\text { marcescens }\end{array}$ & $2(4.5 \%)$ & $4(10.8 \%)$ & 0.2835 & $0.42(0.08-2.17)$ & \\
\hline
\end{tabular}


Table 7.

Placentas with histological signs of inflammation and necrotic changes in Group 3

\begin{tabular}{|l|c|c|c|c|c|}
\hline \multicolumn{1}{|c|}{ Group 3 } & $\begin{array}{c}\text { Signs }+ \\
\mathrm{n}=49\end{array}$ & $\begin{array}{c}\text { Signs - } \\
\mathrm{n}=24\end{array}$ & $P$ & $\begin{array}{c}\text { OR } \\
(95 \% \mathrm{CI})\end{array}$ & $\mathrm{R}$ \\
\hline E.coli & $13(26.5 \%)$ & $2(8.3 \%)$ & 0.0707 & $3.18(0.78-12.99)$ & $\begin{array}{c}0.324 \\
P<0.001\end{array}$ \\
\hline Candida spp. & $4(8.2 \%)$ & $6(25.0 \%)$ & 0.0859 & $0.33(0.10-1.05)$ & \\
\hline $\begin{array}{l}\text { Enterococcus } \\
\text { faecalis }\end{array}$ & $25(51 \%)$ & $6(25 \%)$ & 0.0346 & $2.04(0.97-4.3)$ & $\begin{array}{c}0.6483 \\
P<0.001\end{array}$ \\
\hline Str. agalactiae & $6(12.2 \%)$ & $3(12.5 \%)$ & 0.9752 & $0.98(0.27-3.58)$ & \\
\hline $\begin{array}{l}\text { Staph. } \\
\text { epidermidis }\end{array}$ & $11(22.4 \%)$ & $4(16.7 \%)$ & 0.5657 & $1.35(0.48-3.79)$ & \\
\hline $\begin{array}{l}\text { Staph. aureus } \\
\text { (30(61.2\%) }\end{array}$ & $9(37.5 \%)$ & 0.0563 & $1.63(0.93-2.87)$ & $\begin{array}{c}0.358 \\
P=0.009\end{array}$ \\
\hline $\begin{array}{l}\text { Staph. } \\
\text { haemolyticus }\end{array}$ & $7(14.3 \%)$ & $5(20.8 \%)$ & 0.4783 & $0.69(0.24-1.94)$ & \\
\hline $\begin{array}{l}\text { Enterobacter } \\
\text { aerogenes }\end{array}$ & $8(16.3 \%)$ & $7(29.2 \%)$ & 0.2021 & $0.56(0.23-1.36)$ & \\
\hline Acinebacter & $12(24.5 \%)$ & $7(29.2 \%)$ & 0.6688 & $0.84(0.38-1.86)$ & \\
\hline Str. viridans & $8(16.3 \%)$ & $1(4.2 \%)$ & 0.1377 & $3.92(0.52-29.56)$ & \\
\hline $\begin{array}{l}\text { Staph. } \\
\text { warneri }\end{array}$ & $4(8.2 \%)$ & $6(25 \%)$ & 0.0494 & $0.33(0.1-1.05)$ & \\
\hline $\begin{array}{l}\text { Serratia } \\
\text { marcescens }\end{array}$ & $3(6.1 \%)$ & $4(16.7 \%)$ & 0.1506 & $0.37(0.09-1.51)$ & \\
\hline
\end{tabular}

Table 8 .

Placentas with histological signs of pathological immaturity in Group 3

\begin{tabular}{|l|c|c|c|c|c|}
\hline \multicolumn{1}{|c|}{ Group 3} & $\begin{array}{c}\text { Signs }+ \\
\mathrm{n}=48\end{array}$ & $\begin{array}{c}\text { Signs }- \\
\mathrm{n}=25\end{array}$ & $P$ & $\begin{array}{c}\mathrm{OR} \\
(95 \% \mathrm{CI})\end{array}$ & $\mathrm{R}$ \\
\hline E.coli & $13(27.1 \%)$ & $2(8 \%)$ & 0.0555 & $3.39(0.83-13.84)$ & $\begin{array}{c}0.324 \\
P<0.001\end{array}$ \\
\hline Candida spp & $5(10.4 \%)$ & $5(20 \%)$ & 0.2977 & $0.52(0.17-1.63)$ & \\
\hline $\begin{array}{l}\text { Enterococcus } \\
\text { faecalis }\end{array}$ & $15(31.3 \%)$ & $16(64 \%)$ & 0.0072 & $0.49(0.29-0.82)$ & $\begin{array}{c}0.577 \\
P<0.001\end{array}$ \\
\hline Str. agalactiae & $5(10.4 \%)$ & $4(16 \%)$ & 0.4911 & $0.65(0.19-2.21)$ & \\
\hline $\begin{array}{l}\text { Staph. } \\
\text { epidermidis }\end{array}$ & $9(18.8 \%)$ & $6(24 \%)$ & 0.5983 & $0.78(0.31-1.95)$ & \\
\hline Staph. aureus & $32(66.7 \%)$ & $7(28 \%)$ & 0.0017 & $2.38(1.23-4.61)$ & $\begin{array}{c}0.713 \\
P<0.001\end{array}$ \\
\hline $\begin{array}{l}\text { Staph. } \\
\text { haemolyticus }\end{array}$ & $7(14.6 \%)$ & $5(20 \%)$ & 0.5535 & $0.73(0.26-2.06)$ & \\
\hline $\begin{array}{l}\text { Enterobacter } \\
\text { aerogenes }\end{array}$ & $7(14.6 \%)$ & $8(32 \%)$ & 0.0805 & $0.46(0.19-1.11)$ & \\
\hline Acinebacter & $12(25 \%)$ & $728 \%)$ & 0.7816 & $0.89(0.4-1.98)$ & \\
\hline Str. viridans & $8(16.7 \%)$ & $1(4 \%)$ & 0.1183 & $4.17(0.55-31.47)$ & $\begin{array}{c}0.256 \\
P=0.057\end{array}$ \\
\hline $\begin{array}{l}\text { Staph. } \\
\text { warneri }\end{array}$ & $5(10.4 \%)$ & $5(20 \%)$ & 0.2585 & $0.52(0.17-1.63)$ & \\
\hline $\begin{array}{l}\text { Serratia } \\
\text { marcescens }\end{array}$ & $3(6.3 \%)$ & $4(16 \%)$ & 0.1794 & $0.39(0.09-1.61)$ & \\
\hline
\end{tabular}

Table 9.

Placentas with histological signs of PI and hypertension in Group 3

\begin{tabular}{|l|c|c|c|c|c|}
\hline \multicolumn{1}{|c|}{ Group 3} & $\begin{array}{c}\text { Signs }+ \\
\mathrm{n}=48\end{array}$ & $\begin{array}{c}\text { Signs - } \\
\mathrm{n}=25\end{array}$ & $P$ & $\begin{array}{c}\text { OR } \\
(95 \% \mathrm{CI})\end{array}$ & $\mathrm{R}$ \\
\hline E.coli & $14(29.2 \%)$ & $1(4 \%)$ & 0.0116 & $7.29(1.02-52.3)$ & $\begin{array}{c}0.493 \\
P<0.001\end{array}$ \\
\hline Candida spp. & $9(18.8 \%)$ & $1(4 \%)$ & 0.0820 & $4.69(0.63-34.94)$ & $\begin{array}{c}0.304 \\
P=0.001\end{array}$ \\
\hline $\begin{array}{l}\text { Enterococcus } \\
\text { faecalis }\end{array}$ & $24(50 \%)$ & $7(28 \%)$ & 0.0711 & $1.79(0.9-3.56)$ & $\begin{array}{c}0.277 \\
P=0.012\end{array}$ \\
\hline Str. agalactiae & $5(10.4 \%)$ & $4(16 \%)$ & 0.4911 & $0.65(0.19-2.21)$ & \\
\hline $\begin{array}{l}\text { Staph. } \\
\text { epidermidis }\end{array}$ & $9(18.8 \%)$ & $6(24 \%)$ & 0.5983 & $0.78(0.31-1.95)$ & \\
\hline $\begin{array}{l}\text { Staph. aureus } \\
32(66.7 \%)\end{array}$ & $7(28 \%)$ & 0.0017 & $2.38(1.23-4.61)$ & $\begin{array}{c}0.372 \\
P=0.001\end{array}$ \\
\hline $\begin{array}{l}\text { Staph. } \\
\text { haemolyticus }\end{array}$ & $7(14.6 \%)$ & $5(20 \%)$ & 0.5535 & $0.73(0.26-2.06)$ & \\
\hline $\begin{array}{l}\text { Enterobacter } \\
\text { aerogenes }\end{array}$ & $7(14.6 \%)$ & $8(32 \%)$ & 0.0805 & $0.46(0.19-1.11)$ & \\
\hline Acinebacter & $16(33.3 \%)$ & $3(12 \%)$ & 0.0487 & $2.78(0.89-8.64)$ & $\begin{array}{c}0.309 \\
P=0.002\end{array}$ \\
\hline Str. viridans & $8(16.7 \%)$ & $1(4 \%)$ & 0.1183 & $4.17(0.55-31.47)$ & \\
\hline $\begin{array}{l}\text { Staph. } \\
\text { warneri }\end{array}$ & $6(12.5 \%)$ & $4(16 \%)$ & 0.6798 & $0.78(0.24-2.52)$ & \\
\hline $\begin{array}{l}\text { Serratia } \\
\text { marcescens }\end{array}$ & $5(10.4 \%)$ & $2(8 \%)$ & 0.7393 & $1.3(0.27-6.24)$ & \\
\hline
\end{tabular}

In general, a strong relationship was found between the presence of pathological changes in placentas and the presence of opportunistic flora in women $(\mathrm{R}=0.722, P<0.001)$. The risk of developing pathological changes in the placenta increased by more than 6 times $(\mathrm{OR}=6.14,95 \% \mathrm{CI}=2.32-9.48)$ with the presence of opportunistic flora. Binary logistic regression analysis made it possible to obtain a mathematical model that reliably identifies significant bacteria, the presence of which allows us to predict the revealed changes in the structures of placentas (Table 10-12).

Table 10.

Mathematical model for placentas with signs of inflammation and necrotic changes

Logistic regression (logit) Dep. Var: placentas with signs of inflammation and necrotic changes. Loss: Max likelihood (MS-err. scaled to 1) Final loss: 99.064595337 Chi-square (3) $=69.181 P=.00000$

\begin{tabular}{|l|c|c|c|c|}
\hline & Const.B0 & E.coli - & Staph. aureus & Acinebacter \\
\hline Estimate & 0.2001 & -1.5361 & -1.672 & -2.1480 \\
\hline Standard Error & 0.2144 & 0.4014 & 0.79112 & 0.771 \\
\hline $\mathrm{t}(228)$ & 0.93308 & -3.826 & -2.1134 & -2.783 \\
\hline P-value & 0.3517 & 0.0001 & 0.03564 & 0.0058 \\
\hline -95\%CL & -0.222 & -2.3271 & -3.2308 & -3.668 \\
\hline +95\%CL & 0.6226 & -0.7451 & -0.11314 & -0.627 \\
\hline Wald's Chi-square & 0.870 & 14.643 & 4.46662 & 7.749 \\
\hline P-value & 0.3507 & 0.00013 & 0.03457 & 0.0053 \\
\hline Odds ratio (unit ch) & 1.2215 & 0.215 & 0.18787 & 0.116 \\
\hline -95\%CL & 0.8005 & 0.0975 & 0.0395 & 0.025 \\
\hline +95\%CL & 1.863 & 0.474 & 0.89301 & 0.533 \\
\hline Odds ratio (range) & & 0.215 & 0.18787 & 0.1167 \\
\hline -95\%CL & & 0.097 & 0.039 & 0.025 \\
\hline +95\%CL & & 0.474 & 0.893 & 0.533 \\
\hline
\end{tabular}


Classification of Cases OR: 40.04 Perc. correct: 84.14\%

\begin{tabular}{|c|c|c|c|}
\hline & Pred. - 1 & Pred. - 0 & Percent - Correct \\
\hline 1 & 127 & 7 & 94.78 \\
\hline 0 & 29 & 64 & 68.82 \\
\hline
\end{tabular}

Mathematical model for placentas with signs of of inflammation and necrotic changes: sensitivity $-77.27 \%$, specificity-78.95\%, and accuracy $-77.97 \%$

\section{Table 11.}

Mathematical model for placentas with signs of pathological immaturity

Logistic regression (logit) Dep. Var: placentas with signs of pathological immaturity. Loss: Max likelihood (MS-err. scaled to 1) Final loss: 110.43963104 Chi-square 3) $=90.738 P=0.0000$

\begin{tabular}{|l|c|c|c|c|}
\hline & Const.B0 & $\begin{array}{c}\text { Staph. } \\
\text { aureus }\end{array}$ & $\begin{array}{c}\text { Candida } \\
\text { spp. }\end{array}$ & $\begin{array}{c}\text { Staph. } \\
\text { epidermidis }\end{array}$ \\
\hline Estimate & -1.2375 & 3.8453 & 2.8023 & 1.4181 \\
\hline Standard Error & 0.18690 & 0.62581 & 1.104293 & 0.3792 \\
\hline t(228) & -6.6211 & 6.1445 & 2.5377 & 3.7395 \\
\hline P-value & $<0.0001$ & $<0.0001$ & 0.01182 & 0.0002 \\
\hline -95\%CI & -1.6058 & 2.61222 & 0.62646 & 0.670 \\
\hline +95\%CL & -0.86927 & 5.0784 & 4.97832 & 2.165 \\
\hline Wald's Chi-square & 43.84013 & 37.754 & 6.4400 & 13.98 \\
\hline P-value & $<0.0001$ & $<0.0001$ & 0.01116 & 0.00018 \\
\hline OR (unit ch) & 0.2900 & 46.77496 & 16.484 & 4.1295 \\
\hline -95\%CL & 0.2007176 & 13.629 & 1.8709 & 1.956 \\
\hline +95\%CL & 0.4192561 & 160.52 & 145.23 & 8.718 \\
\hline OR (range) & & 46.774 & 16.484 & 4.129 \\
\hline -95\%CL & & 13.629 & 1.8709 & 1.956 \\
\hline +95\%CL & & 160.52 & 145.2301 & 8.718 \\
\hline
\end{tabular}

Classification of Cases OR: 40.04 Perc. correct: 84.14\%

\begin{tabular}{|c|c|c|c|}
\hline & Pred. - 1 & Pred. - 0 & Percent - Correct \\
\hline 1 & 127 & 7 & 94.78 \\
\hline 0 & 29 & 64 & 68.82 \\
\hline
\end{tabular}

Mathematical model for placentas with signs of pathological immaturity: sensitivity - 94.78\% (95\% CI: 89.61\%-97.45\%), specificity - 68.82\% (95\% CI: 58.81\% - 77.33\%), and accuracy $-84.14 \%$ (95\% CI: $78.29 \%-88.64 \%)$

\section{Table 11.}

Mathematical model for placentas with signs of PI and hypertension Model: Logistic regression (logit) Dep. var: placentas with signs of PI and hypertension. Loss: Max likelihood (MS-err. scaled to 1) Final loss: 106.99411981 Chi-square (3) $=100.20 \quad P=0.0000$

\begin{tabular}{|l|c|c|c|c|}
\hline & Const.B0 & $\begin{array}{c}\text { Enterobacter } \\
\text { aerogenes }\end{array}$ & Candida spp. & E.coli - \\
\hline Estimate & 0.5749 & 1.606 & 4.4158 & -2.9758 \\
\hline Standard Error & 0.22071 & 0.618 & 1.10256 & 0.3818 \\
\hline t(240) & 2.60481 & 2.5963 & 4.00511 & -7.7929 \\
\hline p-value & 0.00976 & 0.0100 & $<0.0001$ & $<0.0001$ \\
\hline -95\%CL & 0.14013 & 0.3875 & 2.2439 & -3.7281 \\
\hline +95\%CL & 1.0097 & 2.825 & 6.5878 & -2.2236 \\
\hline Wald's Chi-square & 6.7850 & 6.7412 & 16.04094 & 60.729 \\
\hline P-value & 0.00919 & 0.0094 & $<0.0001$ & $<0.0001$ \\
\hline OR (unit ch) & 1.7769 & 4.9844 & 82.75615 & 0.05100 \\
\hline -95\%CL & 1.1504 & 1.473 & 9.4305 & 0.0240 \\
\hline +95\%CL & 2.744813 & 16.861 & 726.209 & 0.1082 \\
\hline OR (range) & & 4.984437 & 82.75615 & 0.0510 \\
\hline -95\%CL & & 1.47343 & 9.430586 & 0.0240 \\
\hline +95\%CL & & 16.86175 & 726.209 & 0.10821 \\
\hline
\end{tabular}

Classification of Cases OR: 17.60 Perc. correct: $80.62 \%$

\begin{tabular}{|c|c|c|c|}
\hline & Pred. - 1 & Pred. - 0 & Percent - Correct \\
\hline 1 & 95 & 25 & 79.17 \\
\hline 0 & 19 & 88 & 82.24 \\
\hline
\end{tabular}

Mathematical model for placentas with signs of PI and hypertension: sensitivity $-79.17 \%$ (95\% CI: 71.05\%-85.47\%), specificity - 82.24\% (95\% CI: 73.92\%-88.33\%), and accuracy - 80.62\% (95\% CI: $74.28 \%-85.69 \%)$.

\section{Conclusion}

In pregnant women, the growth of opportunistic flora in diagnostically significant titers in the discharge from the cervical canal has a negative effect on the morphological and functional state of the placental complex in SPB. The constructed mathematical models make it possible to determine, with a high degree of certainty, the main bacteria for all the studied groups of pregnant women, thereby identifying the risk group of women at the stage of pregnancy planning, predicting complications and increasing the possibility of delivering a full-term baby.

\section{Competing Interests} interests.

The authors declare that they have no competing

\section{Sources of Funding}

This study was funded by the Ministry of Education and Science of the Russian Federation on the programme to improve the competitiveness of the Peoples' Friendship University of Russia (RUDN University) among the world's leading research and education centres in 2016-20.

\section{References}

1. Morgan TK. Role of the Placenta in Preterm Birth: A Review. Am J Perinatol. 2016;33(3):258-66. doi: 10.1055/s0035-1570379.

2. Feist H, von Kaisenberg C, Hussein K. [Pathoanatomical and clinical aspects of the placenta in preterm birth]. Pathologe. 2017;38(4):248-259. doi: 10.1007/s00292-0160156-x. [Article in German]

3. World Health Organization. Preterm Birth, Fact Sheet. 19 February 2018. https://www.who.int/news-room/fact-sheets/ detail/preterm-birth .

4. Liu L, Oza S, Hogan D, Chu Y, Perin J, Zhu J, et al. Global, regional, and national causes of under-5 mortality in 2000-15: an updated systematic analysis with implications for the Sustainable Development Goals. Lancet. 2016;388(10063):3027-3035. doi: 10.1016/S01406736(16)31593-8.

5. Alexander JM, Gilstrap LC, Cox SM, McIntire DM, Leveno KJ. Clinical chorioamnionitis and the prognosis for very low birth weight infants. Obstet Gynecol. 1998;91(5 Pt 1):725-9.

6. Romero R, Espinoza J, Gonçalves LF, Kusanovic JP, Friel $\mathrm{L}$, Hassan $\mathrm{S}$. The role of inflammation and infection in preterm 
birth. Semin Reprod Med. 2007;25(1):21-39.

7. Goldenberg RL, Andrews WW, Goepfert AR, FayePetersen O, Cliver SP, Carlo WA, Hauth JC. The Alabama Preterm Birth Study: umbilical cord blood Ureaplasma urealyticum and Mycoplasma hominis cultures in very preterm newborn infants. Am J Obstet Gynecol. 2008;198(1):43.e1-5. doi: 10.1016/j.ajog.2007.07.033.

8. Orazmuradov AA, Kostin IN, Apresyan SV, Parygina AN, Gavrilova AA, Arakelyan GA, et al. The Results of Microbiological Investigations into Preterm Labor. International Journal of Biomedicine. 2019;9(2):144-149.
9. Doyle RM, Harris K, Kamiza S, Harjunmaa U, Ashorn U, Nkhoma M, et al. Bacterial communities found in placental tissues are associated with severe chorioamnionitis and adverse birth outcomes. PLoS One. 2017:12(7): e0180167. doi: 10.1371/journal.pone.0180167. eCollection 2017.

10. Nedoseykina MS, Mishin AV, Baranovskaya EI. [Features of the morphological structure of placentas from women with preterm birth]. Problemy zdorov'ya i ekologii. 2012;4 (34):7983.[Article in Russian].

11. Milovanov AP. Pathology of the mother-placenta-fetus system. M.:Meditsina, 1999. 